or only minor changes in electrophysiological parameters were noted. The S-enantiomer is therefore responsible for the negative dromotropic effects of racemic gallopamil. The PR interval prolongation was greater after administration of the racemate $(p<0.05)$ due to the enhanced bioavailability of S-gallopamil. No significant changes in blood pressure or heart rate were observed.

Supported by the Robert Bosch Foundation, Stuttgart and Knoll AG, Ludwigshafen.

\title{
References
}

Eichelbaum, M., G. Mikus, and B. Vogelgesang, 1984, Br. J. Clin. Pharmacol. 17, 453.

Gross, A.S., B. Borstel, and M. Eichelbaum, 1989, J Chrom. In Press.

O.th.26.6

\section{Catecholamine and cortisol response to yohimbine infusions in comorbidity of depression and panic attacks}

\author{
Grunhaus, L., Haskett, R.F. and Pande, A.C. \\ Department of Psychiatry, University of Michigan, Ann Arbor, Michigan, 48109-0118, U.S.A.
}

Dysregulation of adrenergic receptors and Hypothalamic-Pituitary-Adrenal (HPA) axis has been repeatedly demonstrated in Major Depressive Disorder (MDD). Plasma catecholamines and plasma cortisol have been one of the most commonly used parameters when exploring this dysregulation. Significant differences across studies have made generalization of these findings difficult. Recent studies suggest that when recurrent panic attacks (Panic Disorder) occur concurrently with an episode of MDD (comorbidily of MDD and Panic Disorder) patients are likely to exhibit more severe psychopathology, greater number of affected relatives, worse treatment outcome, and greater lifelong impairment when compared to patients with MDD aione. It has been proposed that patients with comorbidity of MDD and PD suffer from a combined diathesis, depressive and panic, which may produce characteristic patterns of noradrenergic and HPA dysfunction.

To explore this question further we challenged 13 patients meeting research diagnostic criteria (RDC) for MDD

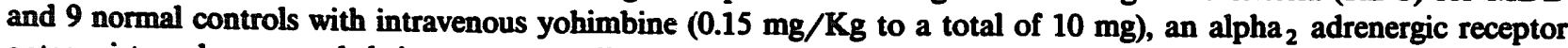
antagonist, and compared their responses. All patients and normal controls had been drug-free for at least 10 days, were on a low VMA diet for the three days preceding the study, spent the preceding night in the Clinical Research Center of the University of Michigan and remained supine for the length of the study. Blood draws were started 60 minutes after the introduction of the IV line. All patients and normal controls undenvent a thorough physical and laboratory examination. Normal controls had no personal or family history of psychiatric disorder. Six patients met the additional criteria of having recurrent panic attacks (meeting RDC-non-hierarchical- Panic Disorder criteria) (MDDpanic group) while the remaining 7 patients, even though having significant anxiety (HRSA $\geq 10$ ) had never experienced panic attacks. We found that patients with MDDpanic had higher resting norepinephrine (NE) levels and pulse. Following the yohimbine infusion patients with MDDpanic showed significantly higher plasma cortisol and NE while plasma epinephrine did not change significantly. Patients with MDDpanic had more intense increases in pulse, anxiety ratings and somatic symptomatology. All patients with MDDpanic experienced a panic attack during the infusion.

Our findings suggest that dysregulation in adrenergic receptors and HPA parameters is more prominent in patients with MDD and recurrent panic attacks. These findings, if supported in larger samples, indicate that accounting for the presence of panic attacks in MDD and of MDD in Panic Disorder patients may improve clinical homogeneity and increase the specificity of the biological findings in these conditions. They also raise several interesting questions. Are patients with comorbidity of MDE and Panic Disorder from a biolcgical perspective closer to patients with Panic Disorder than to $\mathrm{F}$ tients with MDE alone? And finally, do these findings suggest a common genetic background for patients with comoridity of MDE and Panic Disorder and Panic Disorder? 


\title{
References
}

Grunhaus, L. 1988, American Journal of Psychiatry, 145, 1214.

Grunhaus, L., et al., 1989, Clinical Neuropharmacology, 12, 106.

Grunhaus, L., et al., 1990, in-press, Acta Fsychiatrica Scandinavica.

O.th.26.7

\section{Anti-asthmatic drugs: measuring the quality of utilisation}

\author{
Kumana, C.R., So, S.Y., Lauder *, I.J., Ip, M., Lam, W.K. and Kou, M. \\ Department of Medicine, University of Kong Kong, Queen Mary Hospital, Hong Kong \\ and Department of Statistics, University of Hong Kong, Hong Kong
}

The advantages of inhaled as opposed to oral anti-asthmatic drug treatment are widely acknowledged. However, the efficacy of inhaled medication is critically dependent on technique. Anti-asthmatic drug inhalation technique using a Metered Dose Inhaler (MDI) was systematically evaluated in outpatients atrending a busy university run asthma clinic, by a specially trained nurse/practitioner. Inhalation entailed active drug $\boldsymbol{\beta} \beta$-agonist bronchodilator; salbutamol or terbutaline) or control (inert placebo or steroid). Using a-Wright peak flow meter, Peak Expiratory Flow Rate (PEFR) was also determined just before and about 15 minutes after each inhalation procedure.

In all, 74 patients (37 of each sex; mean age 48 years, range 12-82 years) had their inhalation technique scrutinised and relevant characteristics concerning the first 'puff' were recorded. Features regarded as pertinent to inhalation technique were documented as follows:- 1) 73 patients (99\%) removed the MDI cap, 2) 55 (74\%) shook the MDI before use, 3) 36 (49\%) tilted their heads back prior to inhalation, 4) 71 (96\%) actuated the MDI during inspiration, 5) 55 (74\%) achieved a slow deep breath, 6) 20 (27\%) appeared to 'stop' inspiration (at least momentarily) when their MDI was actuated, 7$) 8$ (11\%) engaged in multiple actuation during inspiration, 8) 52 (70\%) held their breath for $<10$ seconds and 9) 58 took their second 'puff' within 30 seconds of completing their first 'puff. Moreover, 49 patients inhaled with the their lips closed round the MDI mouth piece, 23 kept their lips open and 2 held the device at a distance from their mouths.

The mean increase in PEFR ( $\triangle$ PEFR) in 56 patients inhaling $\beta$-agonist was greater than in the 18 controls; mean values $\pm(\mathrm{SD})$ being $19(27)$ and $4(22) \mathrm{L} / \mathrm{min}$ respectively $(\mathrm{p}<0.05)$. Among those inhaling $\beta$-agonist, regression analysis showed that i) tilting back of the head, and ii) actuation appearing to 'stop' inspiration were features associated with the most unsatisfactory responses. When both features were taken together, there was a statistically significant negative association with $\triangle$ PEFR $\left(R^{2}=0.15 ; p<0.02\right)$.

For our population of asthmatic outpatients, education should emphasise the need to avoid i) excessive tilting back of the head, and ii) actuation 'stopping' inspiration.

\section{Ebastine, a non-sedative $\mathrm{H}_{1}$-antihistamine without alcohol interaction}

\author{
Mattila, M.J. and Kuitunen, T. \\ Department of Clinical Pharmacology, University of Helsinki, Paasikivenkatu 4, 00250 Helsinki, Finland
}

Ebastine is a novel $\mathrm{H}_{1}$-antihistamine which poorly penetrates to the brain and effectively blocks $\mathrm{H}_{1}$-receptors in peripheral tissues, without producing important sedation. During its first pass through the liver, ebastine is amply converted to an active metabolite carebastine that does not cumulate (half-life 10-16 hours) during multiple dosing once daily (Rhone-Poulence, data on files). We have studied, double-blindly and crossover, the actions and interactions with alcohol of ebastine on performance. 